\title{
An Advanced Optimization Protocol for Cross Layer Routing in MANET
}

\author{
Bushra Tahseen, P. Suryanarayana Babu
}

\begin{abstract}
Through advancements in communication techniques, there have been significant advances in information technology. Information exchange is captive from infrastructure-based to infrastructure-free techniques. Development in wireless technology and portable computing systems has brought interest in the mobile communication field. The increasing flexibility of people around the network has generated demand for mobile networks such as MANET that can be deployed rapidly and without infrastructure. When users of MANET expect effective communication, seamless reliability is currently crucial across heterogeneous mobile wireless systems. The main challenges in adhoc networks are regular topology changes due to flexibility and limited battery capacity for mobile devices. Depletion of the power source may cause early links in the network to be unavailable. Often, due to frequent breaks in path and affects the performance adversely needed for applications as well as node flexibility. This research paper aims to test and suggest a cross-layer interaction model between transport layer, routing layer, data link layer, and physical layer with power-efficient routing intentions. Using the proposed link prediction model, the article modified the incorporated AODV routing protocol by the link prediction algorithm to predict the accessibility time and even before the connection breaks. The proposed algorithm increases the service quality of the network and NS2 simulator checked the model. The simulation results indicate that the performance of the AODV routing algorithm is much more effective than the current algorithm.
\end{abstract}

Keywords : AODV Routing Protocol, Communication Techniques, MANET, Mobile Wireless Systems, Prediction Algorithm, Wireless Technology.

\section{INTRODUCTION}

A mobile adhoc network (MANET) is a group of wireless nodes that can transfer information without the use of network infrastructure or management. For such networks, there are many prospective uses, including disaster management, security, health care, academia, and industry. Every node in such a network acts as both a host and a router. An elevated mobility is a major restriction with mobile nodes, which usually causes links to be broken and restored. Moreover, the bandwidth of a wireless network is also reduced, and nodes operate on a battery power that is

Revised Manuscript Received on December 30, 2019.

* Correspondence Author

Bushra Tahseen*, Research Scholar, Department of Computer Science and Engineering, Rayalaseema University, Kurnool, Andhra Pradesh, India. Dr. P. Suryanarayana Babu, Professor, Department of Computer Science and Engineering, Rayalaseema University, Kurnool, Andhra Pradesh, India.

(C) The Authors. Published by Blue Eyes Intelligence Engineering and Sciences Publication (BEIESP). This is an open access article under the CC BY-NC-ND license (http://creativecommons.org/licenses/by-nc-nd/4.0/)

ultimately depleted. Therefore, developing a portable adhoc network is extremely difficult, but there are clear prospects for this technology to handle potential communication protocols. A wireless adhoc network works on the concept of one-hop neighbor node broadcasting, in which an origin node transmission signal propagates to all neighboring nodes within its area of communication [1]. Problems associated with transmission power are a common feature that affects the operation of wireless ad hoc networks. The failure to maintain a consistent transmitting capability degrades the variety of transmission and signal power, disputing the efficiency of adhoc wireless networks.

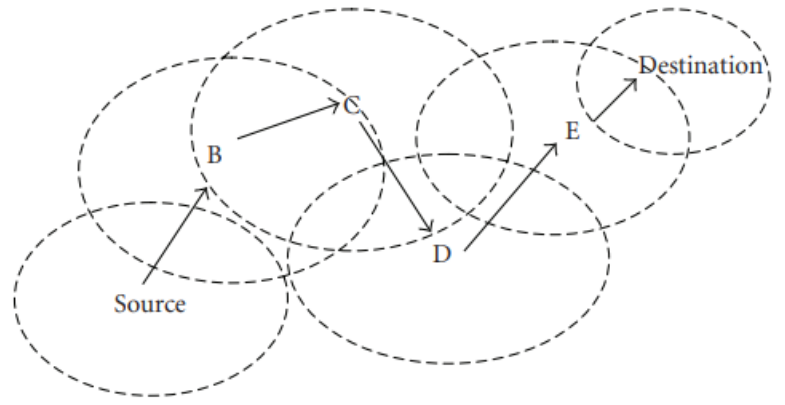

Fig. 1. Example of Adhoc Networking [18].

The presence of the node influences the rate of transmission power on the transport layer and causes congestion of the network. In these networks, TCP-supported congestion control was unreliable, well-known transportation protocols such as UDP were unreliable, as there was no congestion detection mechanism provided. And TCP-backed controls were unreliable [2-3]. Transmission-related issues can affect all stack layers, including: I long delays, (ii) packet losses, and (iii) low performance, from physical to transport.

Previously, the development of adhoc network protocols was based primarily on the layered approach. In layered development, protocol or algorithm designers or implementers concentrate on a single layer without considering the remaining stack parameters [4]. Nonetheless, this has usually led to sub-optimal performance of the program. To solve this, the" cross-layer" approach has been discovered in wireless adhoc networks to tackle energy-based transmission issues. The cross-layer layout deviates from the traditional approach to network design in which each layer of the stack would be designed for independent operation.

\section{LITERATURE REVIEW}

This chapter provides literature review in the cross-layer strategy field specifically aimed at identifying considerations for routing nodes from source to destination without any performance barriers such as power consumption and delay time. 
The research paper literature review was performed with a view to more concise and detailed literature representation.

The effective MANET studies with Cross Layer protocol are given in [8]. One of the most important components of the TCP / IP system pile is the reliability as well as the versatility of the transportation mechanism because these elements are directly connected to the communication and application experience of the user. In this study, we analyze specific problems induced by the efficiency of a trustworthy transportation system by rapidly transforming topology within MANETs in addition to classical transportation layer problems. Instead we propose the Wireless Transport Protocol (WTP) — a brand-new lightweight, bandwidth-efficient, and secure cross-layer transportation system that is intensive in adapting its performance to match highly on-demand environments, such as MANETs. Experimental results show that streamlining the system reduces its operation, while cross-layer optimization makes extra specific changes in network and path variations, thus improving transportation method efficiency by as much as 9 percent in different problems as opposed to the standard TCP protocol. In [9] Proposed network cancelation of the MIMO and SIC interface. Recent developments in MIMO degree-of-freedom (DoF) designs allow us to research MIMO in an environment of multi-hop network. On the other hand, successive cancelation of interference (SIC) is an effective method of physical layer used in multi-user detection. Based on the stamina and also the weak points of MIMO DoF as well as SIC, we propose a marriage between these two methods to ensure that both DoF-based interference cancelation (IC) and SIC can help each other as they adhere to: I SIC is used to decode several received signals to save DoF sources in IC, and (ii) DoFIC solves the potential SINR obstacle that SIC may encounter. In this paper, we develop the necessary mathematical designs in a multi-hop cordless network to understand the two concepts. We create a cross-layer optimization structure with joint DoF IC as well as SIC in addition to scheduling and transmitting restrictions. By using the structure on a question of optimizing throughput, we find that SIC and DoF IC will provide significant improvement in productivity through overcoming the limitation of each other.

Interaction between mobile nodes takes place via wireless media in mobile ad hoc networks [11]. The development of ad hoc network protocol, usually based on a conventional" layered approach,' was found to be unsuccessful in addressing issues related to obtaining signal strength (RSS), affecting the physical layer, network layer, and transportation layer. This article suggests a development strategy to improve the cross-layer communication between different layers, namely physical, MAC and network, which deviates from the traditional architecture of the network. The development strategy for Cross-Layer Power Control (CLPC) would help improve the power of transmission by combining RSS values and finding an efficient path between source and destination. This cross-layer development approach was evaluated by simulation (NS2 simulator [10]) and its performance over AODV was found to be higher. The infrastructure-less and lively design of wireless ad hoc networks demands a fresh set of networking policies to improve the efficiency of service quality (QoS). The availability of QoS in Manets is a major issue. The conventional layered approach is not well suited to ad hoc networks due to high flexibility and vivid topology. This paper applies the Manets DYMO routing protocol's cross-layer development technique using both the standard layered approach and the cross-layer model suggested using Qualnet simulator. The suggested technique is based on four QoS metrics: efficiency, end-to-end latency, distribution ratio of packets, and jitter. Finally, this paper concludes that the cross-layer strategy significantly increases QoS performance in comparison with the layered approach [12]. It is important to improvise equipment with the rapid increase in contact efficiency, and this is a tedious task. There are limitations to older techniques. The cross-layer layout approach meets expectations by separating the doors between layers. This paper describes a network layer AODV routing protocol cross-layer system called the MAC layer distributed planning protocol and the cross-layer Ad hoc On Demand Distance Vector Routing Scheme.

Standard IDS is not enough for these assaults, but the combination of the approach to machine learning could extend IDS capabilities. With machine learning approaches multiple algorithms together can be used to classify the various symptoms associated with the attack. This creates a classification system for decision-making. In terms of low false ratio of rejection and lower consumption of resources, the results indicate their efficiency. Analyzed that DSR does not have an in-built capacity to assess if the loss of packet occurs because of network congestion or node malfunction, resulting in inefficient use of power. They stretch the DSR using cross-layer design to solve this problem. Only when the loss of packet occurs due to failure of node with the cross-layer layout will a route be determined. Simulation outcomes showed that the planned scheme had a 50 percent lower route estimate than DSR. In order to identify the current state of science, this chapter analyzed the literature on performance quality with an intension. The literature obtained on cross-layer approaches has spread across different domains such as MANET, routing protocols, routing algorithm, delay time identification, delay time and power consumption performance optimization, etc. Some research articles identified specific quality factors for performance degradation.

\section{AD HOC ROUTING PROTOCOLS}

\section{A. Proposed Link Prediction Algorithm}

The proposed one in this research is to associate algorithm forecast with AODV routing algorithm implementation. This algorithm uses the strengths of the packet signal to predict links in order to identify the potential for short-term loss of the connection.

\section{B.Implementation of Proposed Protocol}

In the following steps, the power control protocol suggested:

- Transmitter sends an RTS with an optimal power transmission rate including the level of power in the RTS header.

- Decode receiver RTS, detect transmission, observe energy level and calculate optimum transmission power by formula.

- The receiver connects the transmit power to the CTS packet and uses the same optimum CTS transmission energy level. 
- The transmitter then measures the transmission power level and monitors the energy level and determines the optimal transmission power level.

- The transmitter applies the maximum energy transfer to the packet header and sends the DATA packet at this power level.

- The receiver will send ACK at optimum power levels.

\section{Algorithm for Link Prediction}

The present work also suggested a cross-layer architecture for the On-Demand Power Control and Connection Prediction Protocol (ODPCPLP), which offers an alternative to mixed conservation of power and linking accessibility. It combines the effect of maximum transmission capacity with a cross-layer approach to determine the accessibility of the transmitted signal strength based on the existing AODV routing protocol. This technique suggested using optimum transmitting authority to transmit the packets to a nearby with respect to the distance to improve usability of the connection.

The packets transferred power and signal strength received are parameters of cross-layer used to communicate provides the combined power conservation solution and efficient route creation with improved communication accessibility and thus paths between the source and destination. Interactions of Cross-layer between non-adjacent layers are in protocol's stack. It increases efficiency, delivery ratio of packets by predicting in advance breaks in link and initiating the route repair. It also eliminates contact time interruption, overhead routing, point-to-point latency and power consumption by using the interactions of cross-layer. The cross-layer strategy to link accessibility prediction (ODPCPLP) increases the lifespan and capacity of networks and nodes by integrating the effect of optimum transmission power in the transmission of RTS, CTS, DATA and ACK packets and assessing the connection time and further creation of the route prior to connection interruption to meet the application QoS requirements like power control and connection reliability.

\section{SIMULATION RESULTS AND DISCUSSIONS}

\section{A.NS2 Source Generator}

The wireless simulation scenarios are developed by the Network Simulation Source Generator. NSG is a Java-based tool for creating network topologies with appropriate configurations like Agent Type along with packet size and other network parameters like channel type, mac protocol, routing protocol and 9 other parameters.

\section{B. Simulations and Results for Cross-Layer Approach}

Using NS-2 to simulate AODV routing protocol, link AODV (AODVLP) prediction and connection on-demand power control protocol (ODPCPLP) were implemented. In simulation speed, network load (packet generation rate) and nodes count in a given area, we varied three parameters. The complete parameters considered for simulation are listed in Table-I. Numerous simulations are performed with the same parameters and an average of calculated values was taken to decrease the approximation error.

\section{Simulation Parameters} node to boost the battery period and predict signal strength

Table-I. ODPCPLP simulation parameters

\begin{tabular}{|l|l|}
\hline Traffic Pattern & CBR \\
\hline Simulation Time & 900 seconds \\
\hline Simulation Area & $1500 \mathrm{~m}$ by 300m \\
\hline Total Connections & $20,25,30,35,40$ and 45 \\
\hline Velocity & 5 meters/second \\
\hline Packet Size & 512 Bytes \\
\hline Pause Time & 10 seconds \\
\hline Total No. of Nodes & $30,45,60,75$ and 90 \\
\hline
\end{tabular}

\section{Performance Outcomes and Analysis}

The effects of the AODV, AODVLP and ODPCPLP simulation are obtained. The speed of a fixed 50 nodes network size and 10 seconds pause time in figures 2 and 3 varies as 5, 10, 15, 20, 25 and 30 meters per second in separate measurements. Figure 3 shows the average interruption period relation of ODPCPLP, AODVLP and AODV schemes. This reveals that ODPCPLP exhibits the minimal average interruption time when compared to AODV and AODVLP. This is due to ODPCPLP makes use of lower transmission variety and thus simultaneous packet transmission as well as the use of backup direction for path recovery in the event of route failures, resulting in the smallest disruption moment relative to AODV and AODVLP. Nonetheless, AODVLP, AODV and ODPCPLP offer increased a period of average interruption with lower node velocity as faster node flexibility leads to more path unavailability. In turn, a longer interruption period leads to more unavailability of the path.

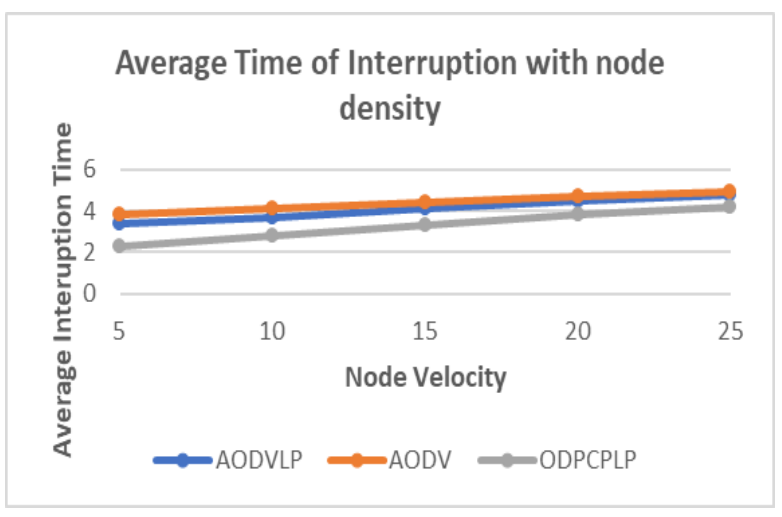

Fig. 2. Average time of interruption vs node velocity

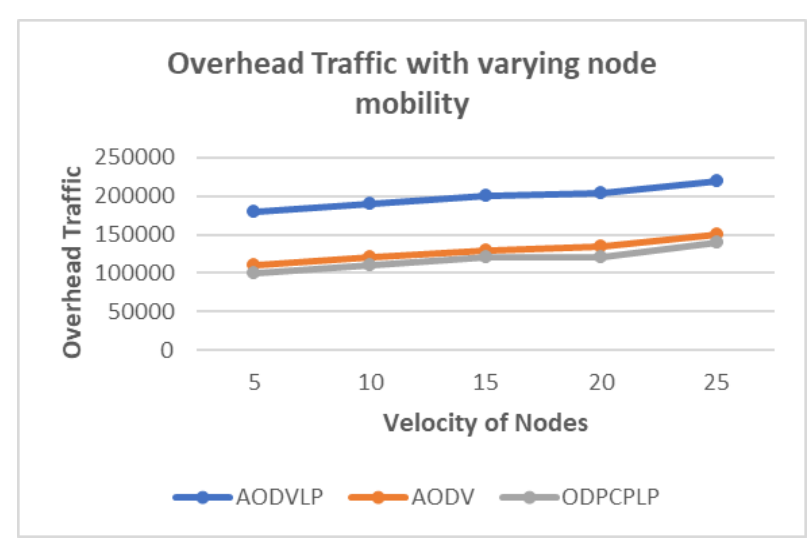

Fig. 3. Node velocity vs Routing overhead 
Figure 3 shows that the overhead packets are less in ODPCPLP than in AODVLP and AODV because, due to a narrower carrier sensing array, more packets are simultaneously transferred in addition to the availability of alternative routes in case of failures because of flexibility of higher nodes. In ODPCPLP, AODV, and AODVLP systems, routing packets overhead improve with increase in node velocity. This is because the speed of the node increases the availability of more route to quickly move nodes. New route discovery overheads thus result in an increase in overhead routing packets. The frequency of packet generation is different when compared with other simulation parameters are held constant for a 50 nodes fixed network size and 10 -second pause time and speed as 5 meters per second in shown in figures 5, 6, 7, 8 and 9. Figure 4 indicates that, due to the route available to increase packet flow, the average interruption period in ODPCPLP is lower than in AODVLP and AODV. In ODPCPLP, the latency time is less as the RTS, CTS, and DATA along with ACK packets are transferred at reduced power with the availability of restore routes in the issue of link failures. When packet generation rate increases, however, AODV, AODVLP and ODPCPLP deliver increased interruption time. Fewer packets would compete at a low speed of 104 packet generation and more packets would compete for transmission at higher network loads and hence more space to interrupt. Therefore, the average interruption period increases with an improvement in the rate of packet generation.

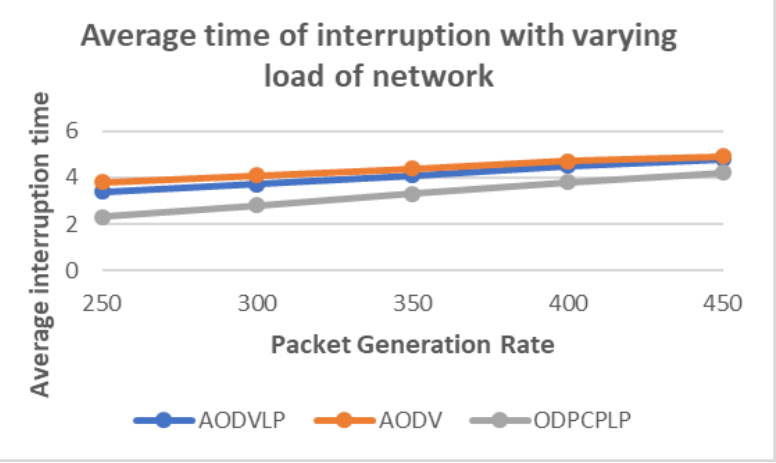

Fig. 4. Packet generation vs Average interruption time

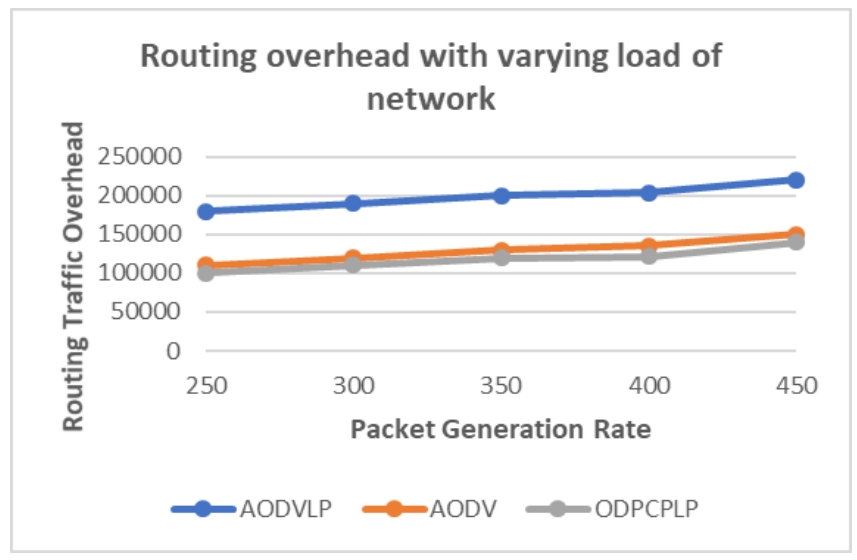

Fig. 5. Packet generation rate vs Routing overhead

Figure 6 shows that less overhead routing packets are generated by the ODPCPLP system compared to AODV and AODVLP systems due to simultaneous packet transmission with respect to reduced transmission power and previous path identification before the link failure, that can prevent packet retransmission in network. The routing overhead packets are growing with an increase in the amount of data packets produced in AODV, AODVLP and ODPCPLP as this enhances contention and collisions. Reduced overhead packets are created by AODV, AODVLP and ODPCPLP at very low packet generation speed. The result shows that the overhead packets are also rising as a higher number of data packets compete for the stream of transmission, generating more overhead packets for packet retransmission by increasing the rate of packet generation.

Figure 7 demonstrates the contrast of AODVLP, AODV and ODPCPLP. This reveals that ODPCPLP achieves the highest throughput when compared with AODV and AODVLP systems. This is due to ODPCPLP use of a smaller variety of carrier sensing when compared to AODV and AODVLP, and large numbers of nodes can be transmitted at the same time. Results indicate that the efficiency of AODVLP is lower than that of AODV. This happens since, before a link failure, alternative paths are also found in advance in ODPCPLP and AODVLP, and a message is sent via alternative path. However, ODPCPLP provides increased throughput as the packet generation speed increases and saturates while keeping the throughput continuous after a point. As with lower packet generation speed, less packets can compete for data transmission and more network loads due to the decrease in power, the count of deferring nodes also decreases and therefore more data can be received per joule, resulting in linear improvements in bandwidth and higher rate of packet generation.

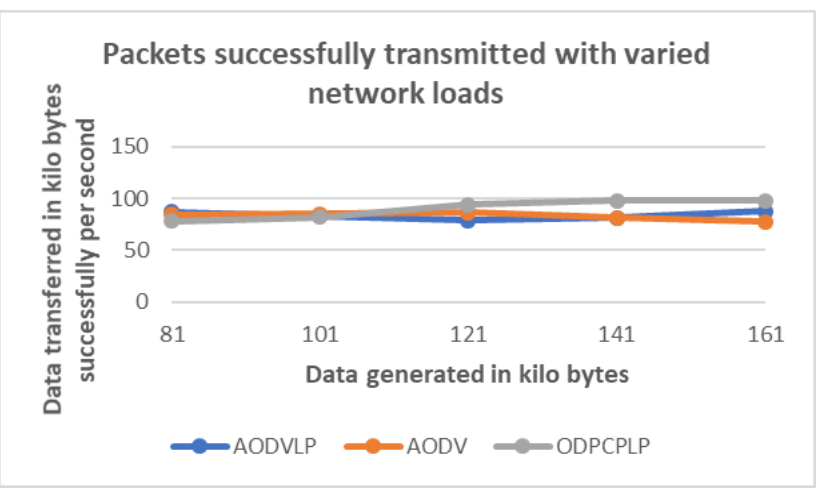

Fig. 6. Packet generation rate vs Throughput

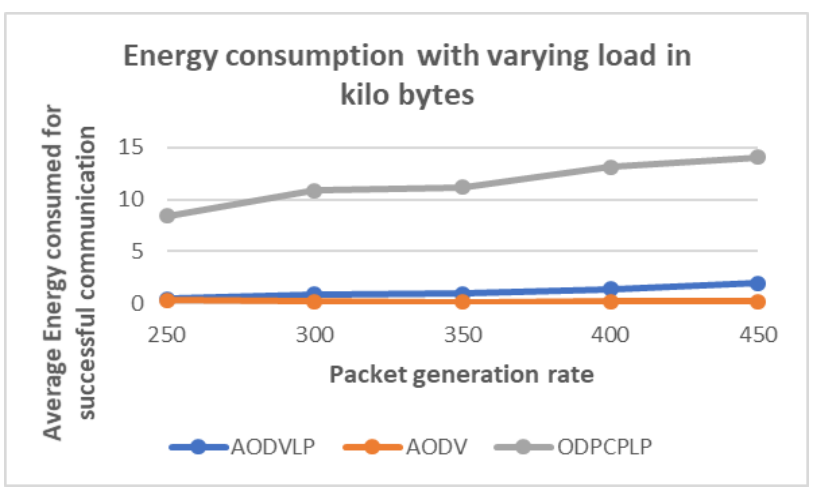

Fig. 7. Average power consumption per 1 kilobyte data communication vs generated packet rate

Published By:

Blue Eyes Intelligence Engineering \& Sciences Publication

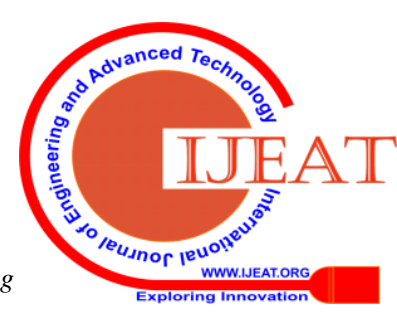


Figure 8 demonstrates power consumption variation by 1 kilobyte of information successfully communicated with an improvement in packet generation speed. The outcome indicates that consumption of power per active 1 kilobyte of information is the lowest in ODPCPLP when compared to AODV and AODVLP. ODPCPLP is less power consumption than other systems because it requires less power to communicate RTS, CTS, and DATA along with ACK packets, and it also observes positive connections and avoids retransmission of packets. Nevertheless, as network load rises, ODPCPLP, AODVLP and AODV provide increased average power consumption as a higher number of packets are generated and contested in the nodes network and as such packets are sent to locations, high energy is consumed in the effective communication of those packets. The size of the network is varied and other relevant simulation parameters are kept constant in figures 8, 9, 10 and 11 with 10 seconds pause time and 5 meters speed per second.

Figure 8 shows that in ODPCPLP, the throughput per node is highest when compared to AODV and AODVLP. This is due to simultaneous transmission is given in the ODPCPLP system due to the use of optimal transmission power, which in case of path failures is lower as well as constructive path exploration and therefore more data. The bandwidth per node decreases in all systems as the number of nodes increases as this creates contention and collisions. Due to lower contention and collisions, the AODV, AODVLP and ODPCPLP provide higher throughput at very low density. Under elevated scale, all three networks have lower throughput because conflicts and collisions are more likely to result from more neighboring nodes.

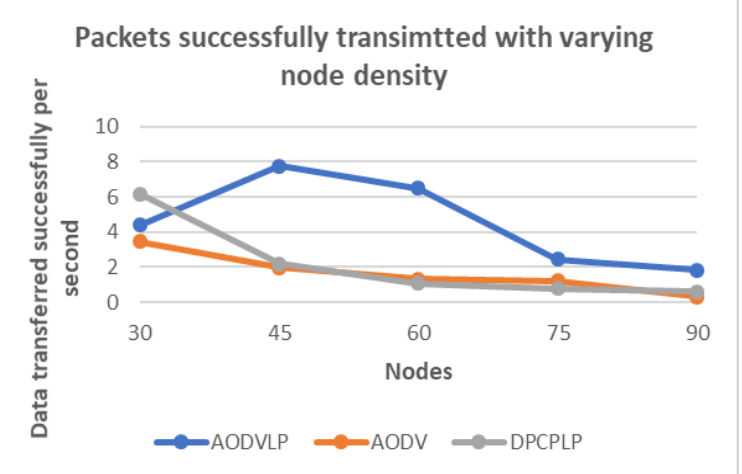

Fig. 8. Number of nodes vs Throughput per each node

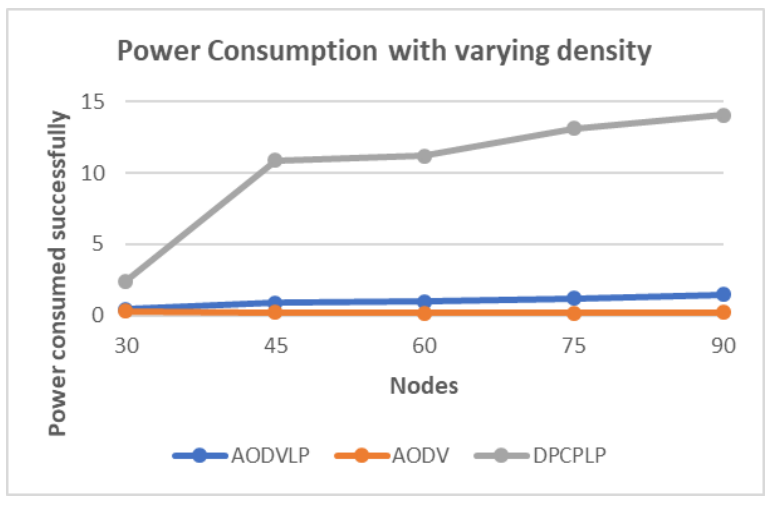

Fig. 9. Power consumption per 1 kilobyte data communication vs number of nodes

Figure 9 indicates that the ODPCPLP protocol saves power, allowing more data packets to be transmitted in less power. The consumption of power increases in every system as the node density rises, there is an increase in contention and collision. But the power consumption of the ODPCPLP in the density variation is least among all the systems making it a good protocol. As the node density increases, Figure 10 indicates differences in the packet distribution ratio. Results show that ODPCPLP is the highest proportion of packet shipping compared with AODVLP and AODV. It happens because simultaneous transmission occurs in ODPCPLP due to channels spatial reuse resulting from decreased packet transmission capacity, alternate paths are discovered in relation to ODPCPLP and AODVLP systems before path failures and a higher number of data is delivered to the target effectively. Nonetheless, as node density increases, ODPCPLP, AODVLP and AODV provide a decreasing delivery ratio as it causes contention and collision because of more competing nodes in the region, reducing the delivery ratio by retransmitting the packets more than once.

The point-to-point lag describes a typical difference between when a data packet is obtained by a request and when the data packet is received at the concerned destination. Figure 11 shows the smallest point-to-point delay in ODPCPLP when compared to AODV and AODVLP as ODPCPLP performs simultaneous packet transmission due to reduced transmission power for RTS, CTS, DATA and ACK as well as previous route discovery in the event of route failure. Due to prior discovery of route in the event of road failures, the point-to-point delay in AODVLP is lower than in AODVLP. The delay is minimal in all low-density systems and increases with complexity as more node density increases the outcome of collusion and contention in the packets retransmission.

Ratio of packet delivery with varying density

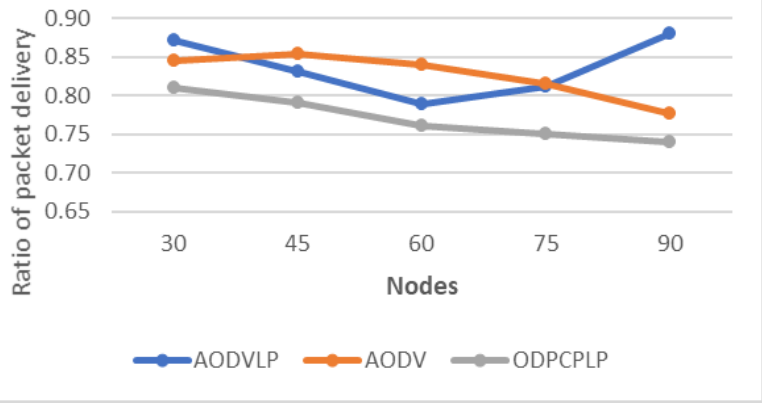

Fig. 10. Packet delivery vs number of nodes

Point-to-Point delay with varying density

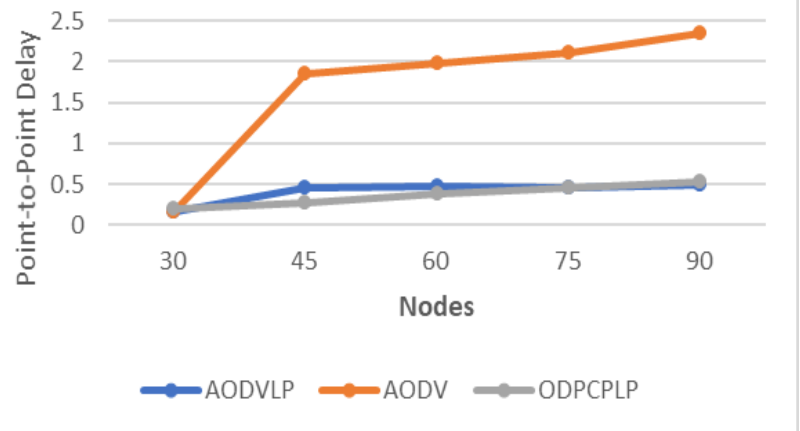

Fig. 11. Point-to-Point delay vs number of nodes

Published By:

Blue Eyes Intelligence Engineering \& Sciences Publication 


\section{An Advanced Optimization Protocol for Cross Layer Routing in MANET}

\section{CONCLUSION AND FUTURE ENHANCEMENTS}

After reviewing the results obtained from NS-2 network simulator, the findings are better than the initial AODV algorithm alone is the AODV routing algorithm (AODVLP) connection forecast model. AODV (AODVLP) link forecast increases QoS by reducing point-to-point delays, restoring the links before track failures, reducing the average collisions of RTS and rising shipping ratios. The power control protocol (DPCP) on-demand is stronger than the IEEE802.11b std. Lower power usage and higher performance. Cross-layer power control layout and connectivity accessibility in portable adhoc networks (ODPCPLP) works better than AODV with AODVLP and without connection forecast (AODV). Cross-layer Power Control and Connectivity Model (ODPCPLP) improves QoS by increasing point-to-point latency, average time of interruption, overhead routing, power consumption, and enhancing shipping percentage and efficiency.

Some other strategies may also be studied for the relation forecast model. The proposed design can also be included in other existing routing algorithms and contrasted with the findings of the LAR and DSR routing algorithms by comparing the quality of link routing forecast while evaluating with the real-time traffic. Other power management protocol can be implemented to boost network performance. It is also possible to include cross-layer compatibility for other layers to further improve network performance. Therefore, the present simulations can be improved to establish protocols for improved efficiency with other systems.

\section{REFERENCES}

1. Park Seungjin, Yoo Seong-Moo. "An efficient reliable one-hop broadcast in mobile ad hoc networks". AdHocNetw2013;11(1):19-28.

2. Senthil kumaran T, Sankaranarayanan V. "Dynamic congestion detection and control routing in ad hoc networks". J King Saud Univ Comput Inform Sci 2013;25(1):65-175.

3. Senthil kumaran T, Sankaranarayanan V. "Early detection congestion and control routing in MANET". Seventh IEEE and IFIP internationa conference proceedings on wireless and optical communications networks (WOCN 2010). p. 1-5.

4. Foukalas Fotis, Vangelis Gazis, Alonistioti Nancy. "Cross-layer design proposals for wireless mobile networks: a survey and taxonomy". IEEE Commun Surv Tutor 2008;10(1):70-84.

5. M. Maleki, K. Dantu, and M. Pedram, "Power Aware Source Routing Protocol for Mobile Ad Hoc Networks", Proceedings of International Symposium on Low Power Electronics and Design, pp. 72-75, 2002

6. A.Michail and A. Ephremides, "Power Efficient Routing for Connection-oriented Traffic in Wireless Ad-Hoc Networks", Mobile Networks and Applications, Vol. 8, No. 5, pp. 517-533, 2003.

7. S. Ahmed and M. S. Alam, "Performance Evaluation of Important Ad Hoc Network Protocols", EURASIP Journal on Wireless Communications and Networking Volume 2006, Article ID 78645, Pages 1-11.

8. Voitenko and M. Derawi, "Reliable Cross-Layer Data Transport Protocol for MANETs," 2014 IEEE 11th International Conference on Mobile Ad Hoc and Sensor Systems, Philadelphia, PA, 2014, pp. 509-510.

9. B. Jalaeian, Y. Shi, X. Yuan, Y. T. Hou, W. Lou and S. F. Midkiff, "Harmonizing SIC and MIMO DoF Interference Cancellation for Efficient Network-Wide Resource Allocation," 2015 IEEE 12th International Conference on Mobile Ad Hoc and Sensor Systems, Dallas, TX, 2015, pp. 316-323.

10. NS2 Network Simulator. http://www.isi.edu/nsnam/ns/ [accessed 04.11.11.]

11. Sarfaraz Ahmed, T. Senthil Kumaran, S. Syed Abdul Syed, S Subburam, "Cross-Layer Design Approach for Power Control in Mobile Ad Hoc Networks", Egyptian Informatics Journal (2015) 16, pp.1-7.
12. Shivam Pankaj Sonar, "Cross Layer Design and Ad Hoc Networks", Available at: at: https://www.researchgate.net/publication/322104260, 2017, pp. 2-12.

13. Dinesh Chandera , Rajneesh Kumar, "QoS Enabled Cross-Layer Multicast Routing over Mobile Ad Hoc Networks", Internationa Conference on Smart Computing \& Communication (ICSCC) 2017 pp. 215-227.

\section{AUTHORS PROFILE}

Bushra Tahseen, is working as an Assistant Professor in the Department of Computer Science and Engineering. She is completed M.Tech from Jawaharlal Nehru Technological University, Anantapur, Andhra Pradesh and currently perusing PhD from Rayalaseema University, Kurnool. She has published 05 articles in various reputed International Journals in the area of Wireless Sensor Networks and MANET.

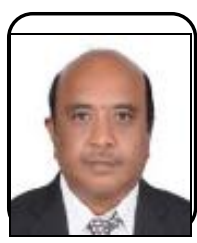

Dr. P. Suryanarayana Babu is a Professor in Department of Computer Science and Engineering in Rayalaseema University, Kurnool, Andhra Pradesh. He has published more than 15 research articles in various International Journals. His area of interest are Mobile Adhoc Networks, Wireless Sensor Networks, Neural Networks, and Information Retrieval. 